\title{
Efecto de cenizas volcánicas del Cordón Caulle sobre parámetros de calidad física en suelos agrícolas del sur de Chile
}

\author{
The effect of volcanic ashes of Cordon Caulle on soil physical quality \\ parameters in agricultural soils of southern Chile
}

\author{
González, R. ${ }^{a}$, Dec, D. ${ }^{a, b}$, Valle, S. ${ }^{a, b}$, Zúñiga, F ${ }^{a, b, c}$, Dörner, J. ${ }^{a, b, *}$ \\ ${ }^{a}$ Instituto de Ingeniería Agraria y Suelos, Universidad Austral de Chile, Valdivia, Chile. \\ ${ }^{b}$ Centro de Investigación en Suelos Volcánicos, Universidad Austral de Chile. \\ ${ }^{c}$ Doctorado en Ciencias Agrarias, Escuela de Graduados, Facultad de Ciencias Agrarias, \\ Universidad Austral de Chile, Valdivia, Chile.
}
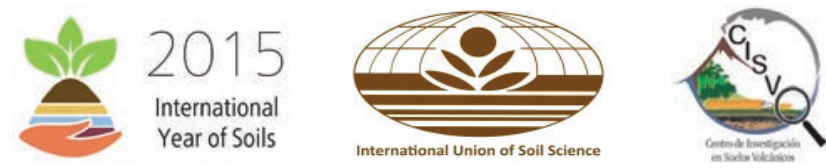

A R T I C LE INFO

Article history:

Received 27.04.2015

Accepted 04.02.2016

Keywords:

Andisols

Volcanic ashes

Soil hydraulic properties

Original Research Article, Special Edition: International Year of Soils (IYS)

Soil Science

*Corresponding author:

Ricardo Gonzalez, José Dörner

E-mail address:

agro tec7@yahoo.es;

josedorner@uach.cl

\section{A B S T R A C T}

Chile is exposed to the constant activity of their volcanoes, which during the eruptions released ashes that had formed soils of south-central Chile (Andisols) during the holocene. The last June of 2011, Cordon Caulle volcano recorded eruptive activity, releasing a great quantity of volcanic materials that were deposited on soils under different land uses, generating environmental problems and uncertainties among farmers for its handling. The aim of this study was to evaluate the effect of the incorporation of these ashes on some physical quality indicators in 6 soil series of southern Chile (five Andisols and one Ultisol). For this, mixes of soil/ashes were used (regarding loads of 0, 5 and $10 \mathrm{~cm}$ of ashes height) to assemble soil cores of $230 \mathrm{~cm}^{3}$. The organic matter content (MO), particle density (Dr), soil texture, $\mathrm{pF}$ curve and air conductivity ( $\mathrm{kl}$ ) were determined. As ashes concentration increased, the sand content increased and, the clay and organic matter contents decreased, inducing an increase in soil particle density. The plant available water increased with the ashes incorporation, which is related to the associated textural changes. $\mathrm{kl}$ increased in all soil series due to incorporation of volcanic ashes, with the exception of the Cudico Series, which suggest the analyses of the effect of wetting and drying cycles on soil structure after the incorporation of volcanic ashes.

\section{RESUMEN}

Chile está expuesto a la constante actividad volcánica, donde el material liberado ha formado los suelos de su zona centrosur (Andisoles) durante el Holoceno. En Junio del 2011 se produjo la erupción del volcán Cordón Caulle, liberando una gran cantidad de ceniza, que fue depositada en terrenos bajo distintos usos, lo que generó problemas ambientales e incertidumbre entre los agricultores debido a que se desconocen los impactos que pueden tener en el suelo. El objetivo de este trabajo fue evaluar el efecto de la incorporación de estas cenizas sobre parámetros de calidad física en 6 series de suelo del Sur de Chile. Para ello, se usaron mezclas suelo/ceniza (0,5 y $10 \mathrm{~cm}$ de altura de acumulación de ceniza sobre el suelo) las que fueron ensambladas en cilindros de un volumen de $230 \mathrm{~cm}^{3}$. Se determinó el contenido de materia orgánica (MO), densidad real (Dr), textura, la curva de retención de agua y conductividad de aire del suelo (kl) en estas mezclas. Al aumentar la concentración de ceniza, aumentó el contenido de arena y disminuyó el contenido de arcillas y MO, lo que implicó un incremento en la Dr. El volumen de agua aprovechable para las plantas y kl aumentaron con el incremento en la carga de cenizas, lo que se relacionó con el cambio en la textura de los Andisoles. Sin embargo, sólo en la Serie Cudico (Ultisol) no se observó un incremento en kl, lo que sugiere la necesidad de analizar el efecto que tienen los ciclos de mojado y secado del suelo en la estructuración de suelos a los que se le han incorporado cenizas volcánicas.

Palabras clave: Andisoles, cenizas volcánicas, propiedades físico hidráulicas del suelo. 


\section{INTRODUCCIÓN}

En el centro sur de Sudamérica, entre los paralelos $36^{\circ}$ y $44^{\circ}$ de latitud sur, existen una serie de volcanes que forman la Cordillera de los Andes y que recorren Chile en toda su extensión. En la actualidad existen 90 volcanes que registran algún tipo de actividad en el país (Sernageomin, 2015). Dentro de estos, se encuentra el volcán Cordón Caulle que junto al volcán Puyehue y otra cumbre denominada Cordillera Nevada, pertenecen a un sistema volcánico conocido en términos vulcanológicos como complejo volcánico Puyehue - Cordón Caulle. Éste se ubica al sur de la región de Los Ríos y en el extremo norte de la región de Los Lagos, con una orientación Noroeste - Sureste entre los $40^{\circ} 32^{\prime} 00^{\prime \prime}$ de latitud Sur y $72^{\circ} 07^{\prime} 00^{\prime \prime}$ de longitud Oeste (Gaitán et al., 2011). De las tres cumbres que componen el complejo, tanto el volcán Puyehue como el Cordón Caulle han presentado actividad eruptiva durante el Holoceno. De estos, solo el volcán cordón Caulle es el que ha presentado un evento eruptivo en el último tiempo. En términos del impacto de estas actividades descritas, se puede mencionar que el macizo liberó una gran cantidad de material piroclástico en forma de cenizas, las que pueden ser trasportadas eólicamente hacia sectores más alejados desde el origen de la erupción siendo depositados finalmente por acción de la gravedad, lo que genera una serie de efectos sobre el ambiente que hasta hoy no han sido totalmente evaluados (Lizcano et al., 2006).

La actividad volcánica desarrollada durante siglos ha permitido la formación y acumulación de grandes cantidades de cenizas volcánicas permitiendo la formación de una importante extensión de suelos derivados de este tipo de materiales (Sanhueza et al., 2011). El proceso de formación de estos suelos, se inicia con la generación de una nube de piroclastos durante una erupción volcánica, conformada por partículas inferiores a $2 \mathrm{~mm}$ conocidas como cenizas volcánicas (Lizcano et al., 2006). A través de diversos procesos de alteración física y química (disolución, lixiviación y precipitación de compuestos), los minerales que conforman las cenizas van cambiando de forma, tamaño y porosidad. La influencia de estos procesos está controlada por condiciones climáticas y por el efecto del tiempo. Las condiciones climáticas determinan la presencia de fluidos disponibles para las reacciones químicas y su tasa de ocurrencia, además de la migración de compuestos y procesos de erosión, entre otros (Towsend, 1985; Chadwick et al., 2003). El tiempo gobierna la secuencia para la síntesis de minerales secundarios y la distribución del tamaño de las partículas (Lizcano et al., 2006), condiciones que de acuerdo al clima templado húmedo del centro sur de Chile han permitido la formación de alofán (Besoaín y Sepúlveda, 1985; Bertrand y Fagel, 2008).
Se estima que el área total cubierta por suelos derivados de cenizas volcánicas en el mundo es de aproximadamente 110 millones de ha, que corresponde a menos de $1 \%$ de la superficie total del planeta y más de la mitad de éstos están situados en los trópicos (Ibáñez y Manríquez, 2011). En Chile, estos corresponden aproximadamente al 60\% de los suelos arables (Tosso, 1985), siendo de vital importancia para el desarrollo de actividades silvoagropecuarias del sur del país. Estos suelos poseen propiedades características conocidas como "ándicas", p.ej. retención de fosfatos $\geq 85 \%$ y densidad aparente $<0,9 \mathrm{Mg} \mathrm{m}^{-3}$ (Shoji et al., 1993), las cuales están dadas por la presencia de minerales tipo aluminio-silicatos no cristalinos de la fracción arcilla, principalmente imogolita-ferrihidrita y complejos humus-aluminio, los que resultan de la meteorización e intemperísmo moderado de depósitos piroclásticos que contienen cantidades significativas de vidrio volcánico en zonas de temperaturas templadas y altos niveles pluviométricos (Lizcano et al., 2006). En ese contexto, la baja densidad aparente y el alto contenido de materia orgánica ( $>12 \%$ ) que presentan estos suelos les permite tener una gran capacidad de almacenamiento (p.ej. agua disponible para las plantas $>20 \%$ ) y conducción de agua (p.ej. conductividad hidráulica saturada $>$ $10 \mathrm{~m} \mathrm{día}^{-1}$ ) que permiten que estos suelos ofrezcan una excelente calidad física para sostener sistemas productivos silvoagropecuarios (Dörner et al., 2013a).

Debido a las actividades silvoagropecuarias desarrollas en el sur de Chile, los estudios en calidad física de suelos derivados de cenizas volcánicas se han enfocado en el efecto del cambio de uso de suelo (Ellies et al., 2000), del manejo de cultivos (Dörner et al., 2012) y praderas (Zúñiga et al., 2015) como así también en el impacto de la intensidad de pastoreo (Dec et al., 2012) sobre el sistema poroso, su funcionamiento y resiliencia. Sin perjuicio de lo anterior, esta zona del país ofrece una posibilidad única a nivel mundial, ya que, debido a la actividad de los volcanes (Besoaín, 1985) estos suelos están expuestos a la acumulación de nuevo material volcánico, cuyo efecto, en conjunto con el potencial impacto sobre el ambiente, requiere ser evaluado. En ese contexto, se han realizado algunos estudios sobre los efectos dañinos que las cenizas provocan en bosques y praderas y sobre las condiciones atmosféricas y la calidad del agua (Hayashi y Okazaki, 2002, 2003; Maglio y Rauque, 2012), como también los problemas que generan las cenizas sobre las actividades humanas y los ecosistemas de la región cercana al lugar del evento eruptivo (Gaitán et al., 2011). Sin embargo, son escasas las evaluaciones sobre los efectos generados por las depositaciones de cenizas sobre la calidad física del suelo (Beke y Sommerfeldt, 1991), lo que es relevante dado que, dependiendo de la altura y granulometría del material acumulado e incorporado al suelo, se pueden producir cambios importantes p.ej. en las propiedades 
hidráulicas del suelo. En ese contexto, se plantea como hipótesis que la incorporación de cenizas volcánicas al suelo implicará un incremento en la capacidad de los suelos de almacenar y conducir agua y aire. El objetivo de este trabajo fue analizar el efecto de la concentración de cenizas en el suelo sobre algunas propiedades físico-hidráulicas en distintos suelos del Sur de Chile.

\section{MATERIALES Y MÉTODOS}

\section{Recolección de muestras}

Muestras disturbadas y no disturbadas (en cilindros metálicos de $230 \mathrm{~cm}^{3}$ ) de suelo fueron recolectadas durante el mes de enero (2012) a una profundidad entre 2-7 cm en 6 series de suelo bajo pradera permanente ubicadas en un transecto desde la Cordillera de los Andes hasta la Cordillera de la Costa (Figura 1):

- Serie Puyehue (PUY; coordenadas: 18G, 701432 S -5494589 E)

- Serie Puerto Fonck (PFO; coordenadas: 18G, 691476 S - 5491149 E)

- Serie Osorno (OSO; coordenadas: 18G, 657978 S - $5505821 \mathrm{E}$ )

- $\quad$ Serie Valdivia (VLD; coordenadas: 18H, 650145
S - 5591396 E)

- Serie Llastuco (LLS; coordenadas: 18H, 670083 S - 5605509 E)

- Serie Cudico (CUD; coordenadas: 18G, 632621 S - 5508166 E)

Las cenizas utilizadas en este estudio fueron recolectas en un sector cercano al evento eruptivo (aproximadamente $8 \mathrm{~km}$ en línea recta al volcán cordón Caulle; 18G, 747874 S - 5522910 E; ver Foto 1) aproximadamente 2 meses después del inicio de la erupción (4 de Junio del Año 2011). Una descripción general de las series de suelo y de las cenizas se presenta en el Cuadro 1.

\section{Preparación de muestras de suelos con distintas concentraciones de cenizas}

\section{Cálculo de la concentración de cenizas en suelo}

Con la densidad aparente (Da) obtenida en cada una de las series de suelo y la ceniza (Cuadro 1) se determinó el peso de suelo considerando 1 ha de suelo con 20 cm de profundidad (Ec. 1). Luego se determinó el peso de la ceniza considerando una carga (altura) de 5 y 10 cm de ceniza. Finalmente, se determinó la relación entre el peso de la ceniza y peso del suelo (Ec. 2), tal como indica en las siguientes ecuaciones:

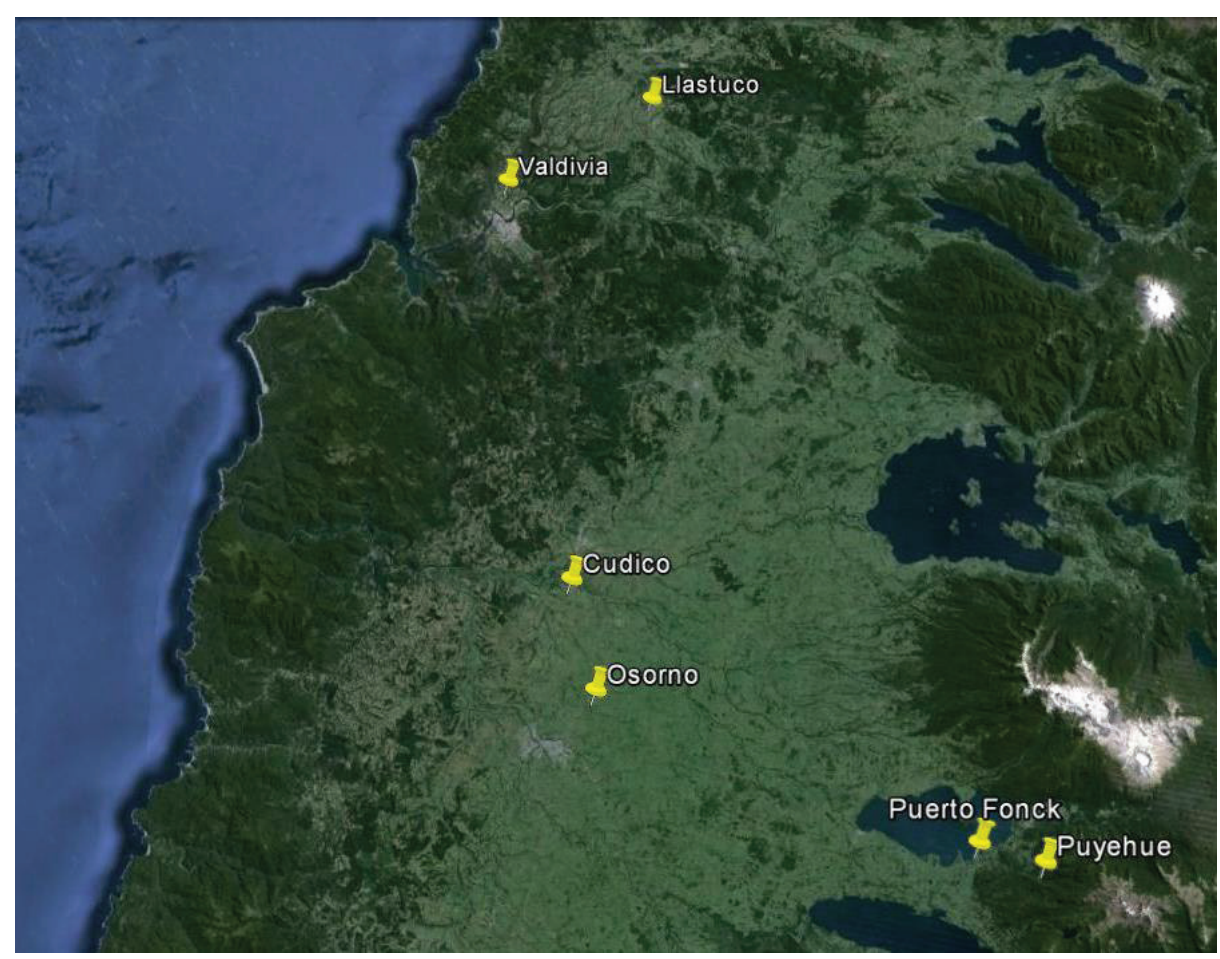

Figura 1. Mapa satelital con ubicación de las series de suelo utilizadas en este estudio. Fuente: Google Earth Pro (2013).

Figure 1. Satellite map with the location of soil series used in this study. Source: Google Earth Pro (2013). 
Cuadro 1. Caracterización general de las series de suelo y cenizas volcánicas estudiadas. Se presentan los valores promedio \pm 1 error estándar.

Table 1. General characterization of studied soil series and volcanic ashes. Mean values \pm 1 standard error are presented.

\begin{tabular}{|c|c|c|c|c|c|c|c|c|c|}
\hline Serie & Textura & $\begin{array}{c}\text { Clasificación } \\
\text { USDA }\end{array}$ & Arena & Limo & Arcilla & MO & Dr & Da & PT \\
\hline & & & \multicolumn{4}{|c|}{ - } & \multicolumn{2}{|c|}{--- $\left[\mathrm{Mg} \mathrm{m}^{-3}\right]-\cdots$} & [\%] \\
\hline PUY** & A-AL & TD & $48,74 \pm 2,69$ & $40,25 \pm 2,91$ & $11,01 \pm 0,23$ & $9,50 \pm 0,05$ & $2,06 \pm 0,01$ & $0,58 \pm 0,00$ & $75,04 \pm 0,43$ \\
\hline PFO & F-FL & PM & $32,69 \pm 1,52$ & $58,11 \pm 1,76$ & $9,20 \pm 0,31$ & $7,21 \pm 0,12$ & $2,14 \pm 0,01$ & $0,74 \pm 0,01$ & $67,62 \pm 0,39$ \\
\hline Oso & FL & $\mathrm{TH}$ & $14,32 \pm 0,17$ & $68,79 \pm 1,18$ & $16,89 \pm 1,04$ & $8,33 \pm 0,10$ & $2,03 \pm 0,01$ & $0,76 \pm 0,01$ & $64,14 \pm 0,58$ \\
\hline VLD & FL & DH & $17,22 \pm 0,62$ & $64,09 \pm 0,73$ & $18,70 \pm 0,25$ & $6,86 \pm 0,12$ & $2,18 \pm 0,01$ & $0,77 \pm 0,01$ & $68,7 \pm 0,27$ \\
\hline LLS & FL & $\mathrm{AH}$ & $15,14 \pm 3,41$ & $56,25 \pm 3,52$ & $28,60 \pm 0,30$ & $7,68 \pm 0,12$ & $2,25 \pm 0,00$ & $0,90 \pm 0,01$ & $65,93 \pm 0,73$ \\
\hline CUD & $\mathrm{Fa}$ & $\mathrm{TH}^{*}$ & $17,94 \pm 0,79$ & $53,34 \pm 1,15$ & $38,65 \pm 1,73$ & $2,05 \pm 0,02$ & $2,35 \pm 0,04$ & $1,29 \pm 0,02$ & $53,80 \pm 0,68$ \\
\hline CEN & ----- & ----- & $53,7 \pm 0,55$ & $43,39 \pm 0,55$ & $2,91 \pm 0,00$ & ------- & $2,41 \pm 0,01$ & $1,03 \pm 2,69$ & --------- \\
\hline
\end{tabular}

FUENTE: CIREN 2011; **: Cook et al., 1985

A: Arenoso; AL: Areno limoso; F: Franco; FL: Franco limoso; Fa: Franco arcilloso; TD: Typic Dystransept; PM: Pachic Melanudands: TH: Typic Hapludands; AH: Acrudoxic Hapludands; TH*: Typic Hapludults.

$$
\begin{gathered}
P_{c ; s}=D a_{c ; s} \times V_{c ; s} \quad[\mathrm{Mg}] \\
C_{c / s}=\left[\frac{P c}{P S}\right] \times 100 \quad[\%]
\end{gathered}
$$

Dónde: $\mathrm{P}_{\mathrm{s} ; \mathrm{c}}$ : Peso (suelo seco o ceniza) $[\mathrm{Mg}] ; \mathrm{Da}_{\mathrm{sic}}$ : Densidad aparente (suelo o ceniza) $\left[\mathrm{Mg} \mathrm{m}^{-3}\right] ; \mathrm{V}_{\mathrm{s} ; \mathrm{c}}$ : Volumen (suelo o ceniza) $\left[\mathrm{m}^{3}\right] ; \mathrm{C}_{\mathrm{c} / \mathrm{s}}$ : Porcentaje de ceniza en suelo según altura de carga de ceniza (5 o $10 \mathrm{~cm}$ ). Finalmente, esto permitió conocer el porcentaje de partición de las cenizas por unidad de peso de suelo para cada carga (altura) de ceniza considerada, lo que se denominó concentración de ceniza en mezcla ceniza - suelo $\left(\mathrm{C}_{\mathrm{c} / \mathrm{s}}\right)[\%]$.

\section{Ensamblaje de muestras}

Para la preparación de las muestras se utilizó un cilindro con un diámetro $(0,056 \mathrm{~m})$ y altura $(0,072 \mathrm{~m})$ estandarizados. Además, se consideró una densidad aparente de $0,7 \mathrm{Mg} \mathrm{m}^{-3}$ para los Andisoles y $1,1 \mathrm{Mg} \mathrm{m}^{-3}$ para el Ultisol (tomando como referencia valores de densidad aparente publicados en CIREN, 2003 y Dörner et al., 2013a). Se determinó la humedad del suelo y de ambas mezclas con suelo - ceniza a fin de determinar la cantidad de agua a aplicar para alcanzar una humedad del 35\% (bss) considerado óptimo para el armado de muestras en cilindros (Sandoval, 2009). Una vez preparada la mezcla húmeda, se procedió a incorporar el suelo al cilindro en láminas de aproximadamente 1 cm hasta alcanzar la densidad aparente deseada según orden de suelo. Una vez que las muestras fueron ensambladas, y con el fin de obtener una mezcla homogénea entre la ceniza y el suelo, estas se sometieron a dos ciclos de humectación y secado (rango de 0 a $60 \mathrm{hPa}$ ) por medio de ascenso capilar y drenaje en bandejas de arena (60 hPa de tensión). Cada ciclo duró 7 días, con 2 días para la humectación y 5 días para el drenaje posterior. En cada ciclo se determinaron los cambios en el volumen de agua y suelo.

\section{Análisis de laboratorio}

\section{Textura, densidad real y contenido de materia orgánica de los suelos y mezclas}

Las muestras de suelo, incluidas las dos mezclas de cenizas, fueron secadas al aire y tamizadas a $2 \mathrm{~mm}$. La textura se determinó por el método del hidrómetro (Day, 1965) luego del acondicionamiento de las muestras (Dörner et al., 2013b). La densidad real (Dr) fue determinada por el método del picnómetro usando vacío (Klute, 1986). Para la determinación de materia orgánica (MO) se utilizó el método de combustión húmeda (Sadzawka et al., 2004).

\section{Propiedades físico-hidráulicas los suelos y mezclas}

Para la determinación de la curva de retención de agua (pF), las muestras fueron saturadas por ascenso capilar de agua y posteriormente equilibradas a distintas tensiones de agua $(10,20,30,60,150,330$ y 15000 
hPa). Una vez alcanzado el equilibrio entre la tensión aplicada y el contenido gravimétrico de agua, se registró el peso (Balanza Precisa, precisión 0,01 g) y altura (Vernier Caliper, precisión de 0,01mm) de las muestras. Esto último, para corregir el contenido volumétrico de agua por la deformación que sufren los suelos volcánicos con el secado (Dörner et al., 2010a). A partir de esta información y la determinación de la densidad aparente del suelo, se determinó el contenido volumétrico de agua para cada tensión. Luego de someter las muestras a $330 \mathrm{hPa}$ de tensión, se secaron a temperatura ambiente $\left(20^{\circ} \mathrm{C}\right.$, por 3 días) para posteriormente incrementar la temperatura $30^{\circ} \mathrm{C}$ (por 3 días), $65^{\circ} \mathrm{C}$ (1 día) y finalmente $105^{\circ} \mathrm{C}$ ( 1 día). Durante estas mediciones se registró el peso y altura de las muestras para determinar la curva de contracción (no presentada en este trabajo). A partir de la curva de retención de agua se determinó la distribución de poros en el suelo (Hartge y Horn, 2009).

La determinación de la conductividad de aire (kl) se hizo $60 \mathrm{hPa}$ de tensión, ya que a una mayor tensión de agua ejercida, las muestras sufrían una contracción horizontal que las separaba del cilindro donde se encontraban ubicadas, lo que hacía imposible una medición de kl. Esta propiedad se determinó a partir de las mediciones realizadas en un flujómetro de aire, el cual mide el volumen de aire que fluye verticalmente a través de la muestra en el tiempo. Mayores detalles respecto al análisis se pueden encontrar en Dörner y Horn (2006).

\section{Análisis estadísticos}

Como parte de la caracterización de cada suelo se determinaron estadísticos descriptivos (media, mínimo, máximo y error estándar) para el análisis textural, conductividad de aire, distribución del tamaño de poros, contenido de materia orgánica y densidad real. Se realizó un ANDEVA factorial (suelo y ceniza) y según correspondió un test de Tukey $(\mathrm{p} \leq 0,05)$ para evaluar el efecto de la concentración de ceniza en cada serie de suelo. Debido a que no existió una interacción significativa entre los factores de análisis (suelo y concentración de cenizas) estos se analizaron de forma independiente.

\section{RESULTADOS}

\section{Características de la ceniza utilizada en el estudio}

Del análisis textural (Cuadro 1) se desprende que las cenizas utilizadas en este estudio corresponden a la clase textural franco arenosa con un 53,7\% arena, principalmente arena fina, un $43,4 \%$ de limo y un $2,9 \%$ de arcilla. Por otro lado, la densidad real de las cenizas utilizadas en el estudio es de $2,41 \mathrm{Mg} \mathrm{m}^{-3}$. Al comparar la textura de la ceniza con la de las series de suelo, des- taca que las diferencias en la fracción más gruesa y fina de las partículas aumentan en la medida que las series se distancian de la Cordillera de los Andes: desde PUY hasta CUD, siguiendo la misma secuencia presentada en el Cuadro 1. Lo mismo se aprecia para la densidad aparente.

\section{Efectos de la incorporación de las cenizas volcánicas sobre la fracción sólida del suelo}

En la medida que los suelos se alejan de la Cordillera de los Andes disminuye el contenido de arena y aumenta el contenido de arcilla (Cuadro 1). La incorporación de ceniza aumenta la fracción de arena, encontrándose diferencias estadísticamente significativas para la serie PUY, PFO, VLD y CUD $(\mathrm{p} \leq 0,05)$. Cuando aumenta la concentración de ceniza en la mezcla ceniza - suelo $\left(\mathrm{C}_{\mathrm{c} / \mathrm{s}}\right)$, aumenta la fracción de arena, encontrándose diferencias estadísticamente significativas solo para las serie OSO (Figura 1). En el caso del limo, no se observó una tendencia clara, determinándose una reducción significativa con la incorporación de ceniza y el incremento de $\mathrm{C}_{\mathrm{c} / \mathrm{s}}$ en el caso de las series VLD y OSO, respectivamente. El contenido de arcilla, en general, disminuye al incorporar ceniza para todas las series de suelo y solo en el caso de la serie VLD no se observaron diferencias estadísticamente significativas.

La Dr tiende a aumentar con el incremento en $\mathrm{C}_{\mathrm{c} / \mathrm{s}^{\prime}}$ observándose diferencias estadísticamente significativas para las serie de suelo: PUY, PFO, OSO y VLD (Figura 2).

Finalmente, se observó que la MO disminuye significativamente $(\mathrm{p} \leq 0,05)$ con la incorporación de cenizas al suelo (Figura 3 ).

\section{Efecto de la incorporación de cenizas volcánicas sobre el volumen poroso del suelo y su funcionamiento}

Para estudiar el efecto de la incorporación y aumento de $\mathrm{C}_{\mathrm{c} / \mathrm{s}}$ sobre el volumen de poros del suelo (Figura 4), el análisis se enfocó en los poros de agua útil (PAU), que están directamente relacionados con el volumen de agua aprovechable para las plantas, y los poros de agua inútil (PAI), asociado a la fracción más fina del suelo (Figura 4). La incorporación de cenizas al suelo significó un aumento en el volumen de agua aprovechable para las plantas, esto, sin embargo, fue en distinta magnitud dependiendo de la textura original del suelo y de la diferencia de esta con la textura de la ceniza incorporada. Para la series PUY y VLD se determinó un incremento significativo $(p \leq 0,05)$ en PAU con la incorporación de ceniza al suelo, sin embargo, esta no siguió aumentando con el incremento en $\mathrm{C}_{\mathrm{c} / \mathrm{s}}$. Por otro lado, para las series PFO, OSO y LLS se registró un incremento significativo en la porosidad al considerar tanto la 
incorporación de ceniza como el incremento en $\mathrm{C}_{\mathrm{c} / \mathrm{s}}$. En el caso de la serie CUD se observa un incremento de PAU solo al nivel más alto de $\mathrm{C}_{\mathrm{c} / \mathrm{s}}$. Con respecto los PAI se observa que en general, la incorporación de ceniza disminuye este tipo de porosidad, sin presentarse diferencias estadísticamente significativas para la serie VLD.

La incorporación de cenizas al suelo significó un incremento en la conductividad de aire (kl, Figura 5). Sin embargo, al aumentar $\mathrm{C}_{\mathrm{c} / \mathrm{s}}$ no se observaron mayores diferencias. Lo anterior, no fue observado para el caso de la serie CUD.

\section{DISCUSIÓN}

Las cenizas volcánicas son partículas de tamaño $\leq 2$ mm generadas a partir de la fragmentación del magma y materiales provenientes de una erupción volcánica (Lizcano et al., 2006). Estas partículas pueden permanecer suspendidas en la atmósfera desde días a meses (Riley et al., 2003) hasta ser depositadas sobre la superficie del suelo, vegetación, masas de agua, entre otras. Cabe destacar que las fracciones de arena, limo y arcilla provenientes de una erupción volcánica no tienen relación ni son equivalentes a aquellas fracciones

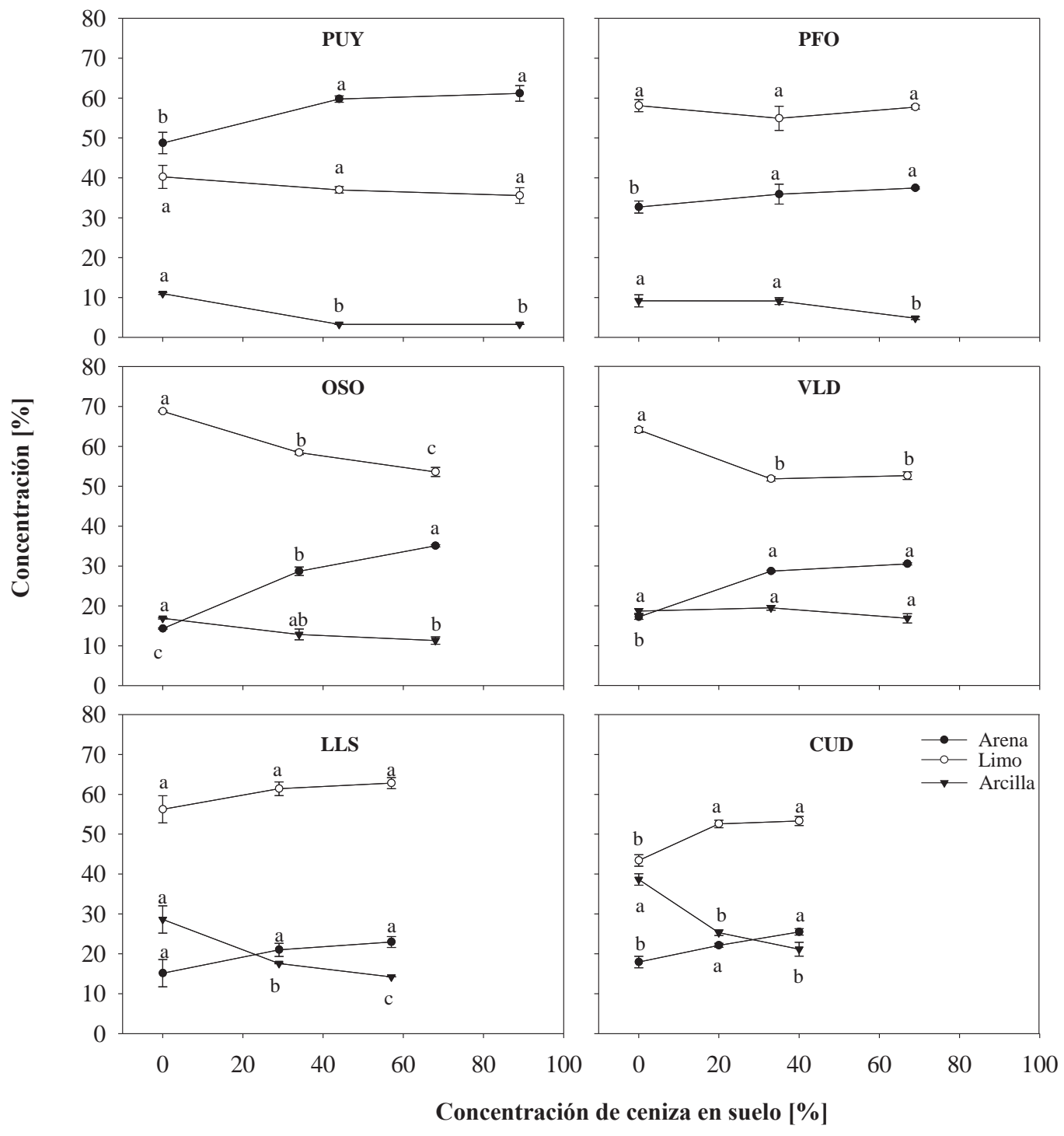

Figura 2. Efecto de la concentración de ceniza/suelo $\left(\mathrm{C}_{\mathrm{c} / \mathrm{s}}\right)$ sobre el contenido de arena, limo y arcilla de las series de suelo. Se presentan valores promedio \pm 1 error estándar $(n=3)$. Letras distintas indican diferencias estadísticamente significativas para las distintas concentraciones de ceniza en una misma fracción textural $(\mathrm{p} \leq 0,05)$.

Figure 2. Effect of ash/soil concentration $\left(\mathrm{C}_{\mathrm{c} / \mathrm{s}}\right)$ on the content of sand, silt and clay of the soil series. Mean values \pm 1 standard error $(n=3)$ are presented. Different letters indicate statistically significant differences for the different concentrations of ash in the same textural fraction $(\mathrm{p} \leq 0,05)$. 


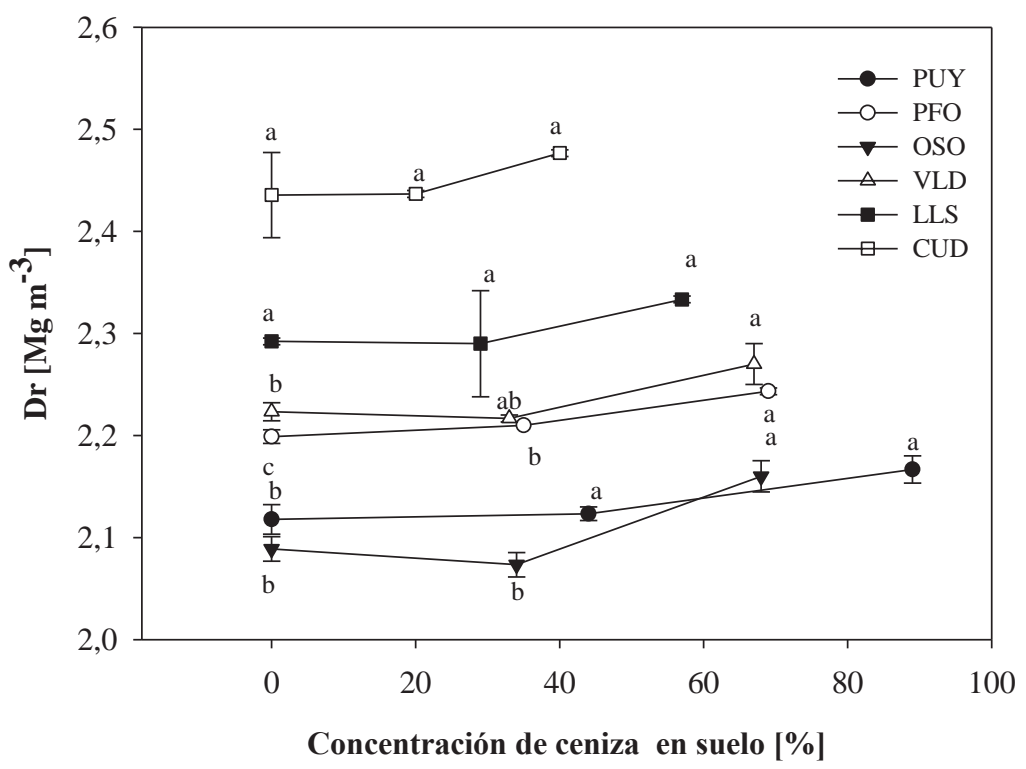

Figura 3. Efecto de la concentración de ceniza/suelo $\left(\mathrm{C}_{\mathrm{c} / \mathrm{s}}\right)$ sobre la densidad real (Dr) de las series de suelo. Se presentan valores promedio \pm 1 error estándar $(\mathrm{n}=3$ ). Letras distintas indican diferencias estadísticamente significativas entre las concentraciones de ceniza para cada serie de suelo $(\mathrm{p} \leq 0,05)$.

Figure 3. Effect of ash/soil concentration $\left(\mathrm{C}_{\mathrm{c} / \mathrm{s}}\right)$ on the particle density (Dr) of the soil series. Mean values \pm 1 standard error $(\mathrm{n}=3)$ are presented. Different letters indicate statistically significant differences between ash concentrations for each soil series $(\mathrm{p} \leq 0,05)$.

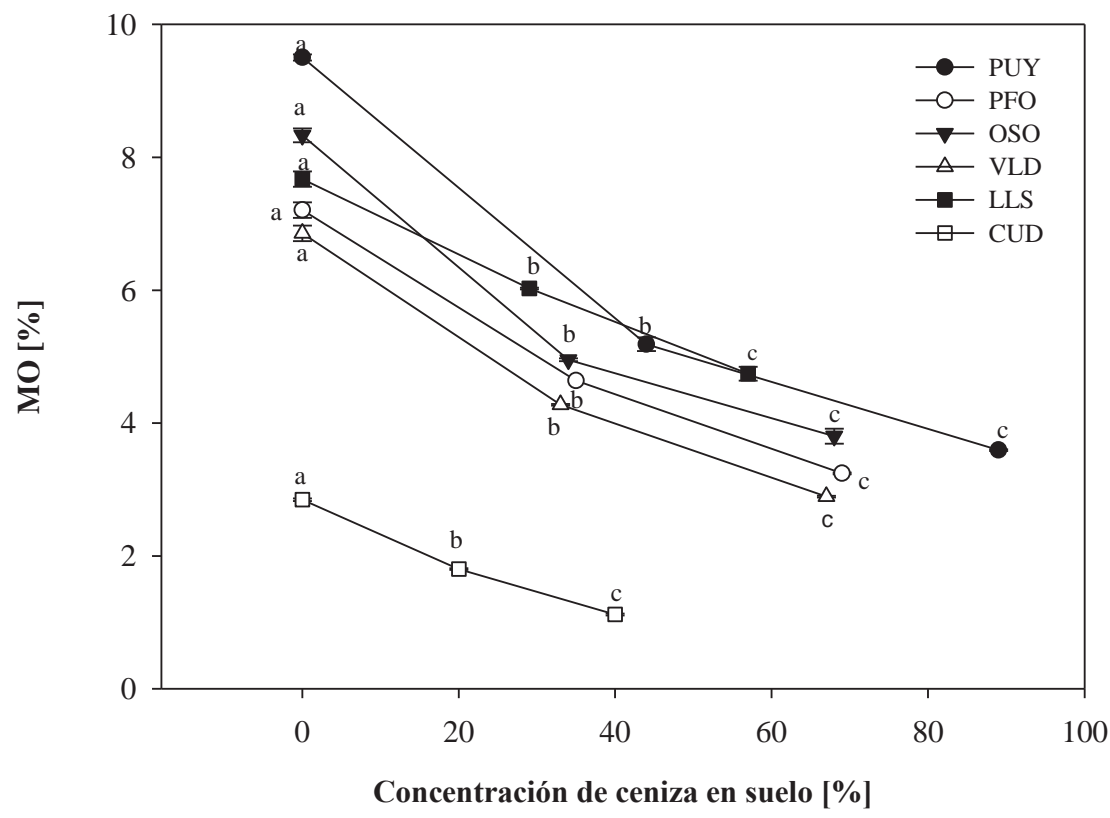

Figura 4. Efecto de la concentración de ceniza/suelo $\left(\mathrm{C}_{c / s}\right)$ sobre el contenido de materia orgánica (MO) de las series de suelo. Se presentan valores promedio \pm 1 error estándar $(\mathrm{n}=3)$. Letras distintas indican diferencias estadísticamente significativas entre las concentraciones de ceniza para cada serie de suelo ( $\mathrm{p} \leq 0,05)$.

Figure 4. Effect of ash/soil concentration $\left(\mathrm{C}_{\mathrm{c} / \mathrm{s}}\right)$ on the organic matter content $(\mathrm{OM})$ of soil series. Mean values \pm 1 standard error $(n=3)$ are presented. Different letters indicate statistically significant differences between the ash concentrations for each soil series $(\mathrm{p} \leq 0,05)$. 

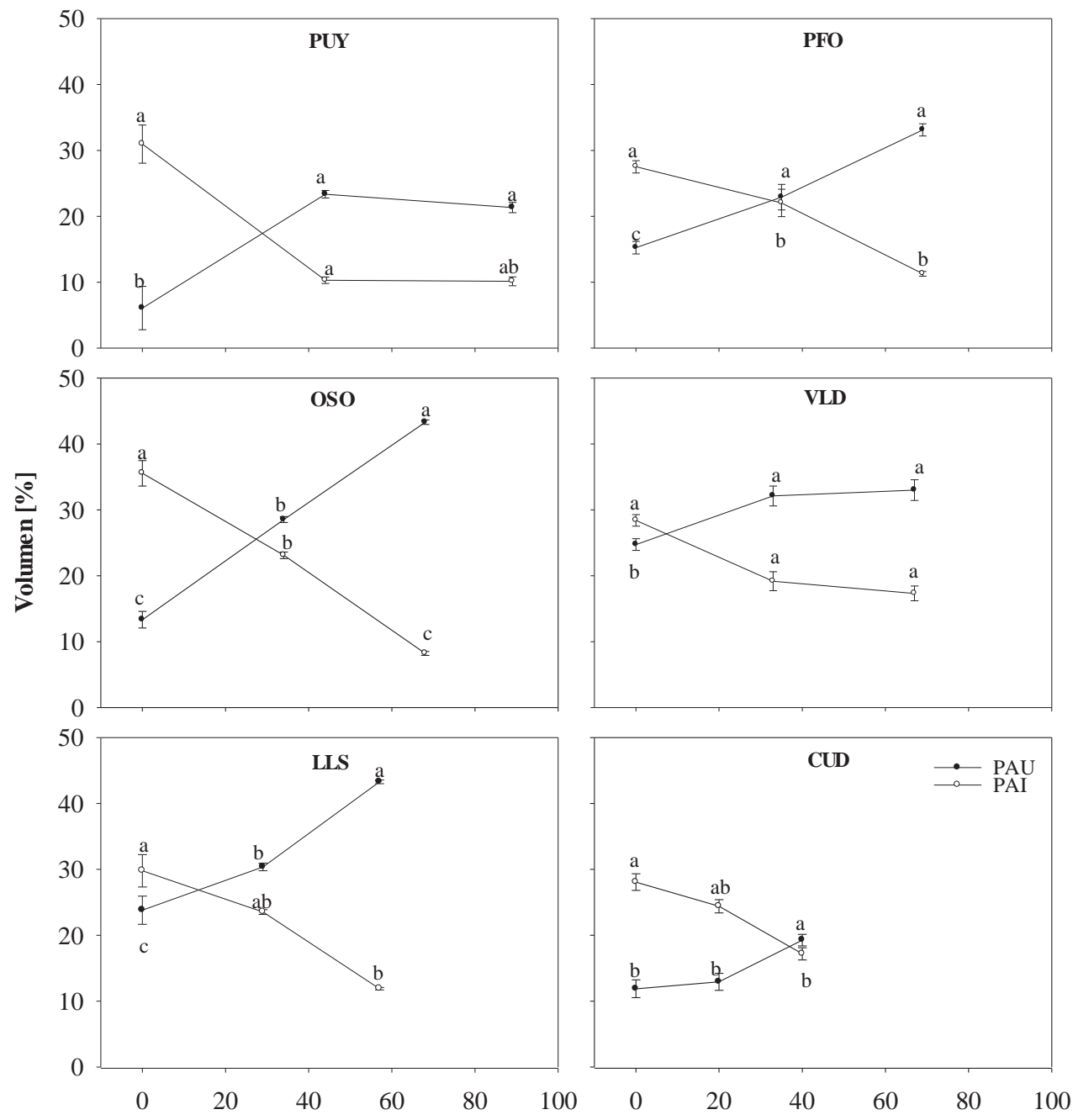

Concentración de ceniza en suelo, $\mathrm{C}_{\mathrm{c} / \mathrm{s}}[\%]$

Figura 5. Efecto de la concentración de ceniza/suelo $\left(\mathrm{C}_{\mathrm{c} / \mathrm{s}}\right)$ sobre el volumen de poros de agua útil (PAU) e inútil (PAI) de las series de suelo. Se presentan valores promedio \pm 1 error estándar $(n=7)$. Letras distintas indican diferencias estadísticamente significativas entre las concentraciones de ceniza para cada serie de suelo $(p \leq 0,05)$.

Figure 5. Effect of ash/soil concentration $\left(\mathrm{C}_{\mathrm{c} / \mathrm{s}}\right)$ on the volume of medium (PAU) and fine (PAI) pores of soil series. Mean values \pm 1 standard error $(n=3)$ are presented. Different letters indicate statistically significant differences between the ash concentrations for each soil series $(\mathrm{p} \leq 0,05)$.

homólogas secundarias que existen en los suelos, salvo la coincidencia en el tamaño (Besoaín et al., 1992).

La morfología y textura de las cenizas varía desde que son emanadas hasta su depositación, lo que finalmente afecta sus propiedades y por ende su respuesta a la incorporación de estas en suelo, por esta razón se hace necesario conocer la textura de las cenizas utilizadas (Cuadro 1). El análisis textural de las muestras ensambladas mostró que, en general, el contenido de arenas aumenta a medida que aumenta la concentración de ceniza en la mezcla; sin embargo, esta respuesta varía dependiendo de la textura inicial y de la cantidad de arcilla que posea el suelo. Lo anterior es relevante dado que el efecto de las cenizas sobre el comportamiento físico del suelo (lo que está asociado al movimiento de agua en el perfil) depende del tamaño de las partículas que forman las cenizas, así, en suelos con partículas más finas las cenizas pueden ayudar a mejorar la infiltración de agua; sin embargo, esto no se observó en suelos con partículas más gruesas, lo que coincide con lo observado por Cruz (2011).

El análisis de densidad real muestra que, aun cuando existe una respuesta a la presencia y al aumento de concentración de ceniza, esta respuesta es muy pequeña e incluso no significativa, a excepción de la serie PFO (Figura 2). Por otro lado, la disminución del conteni- 


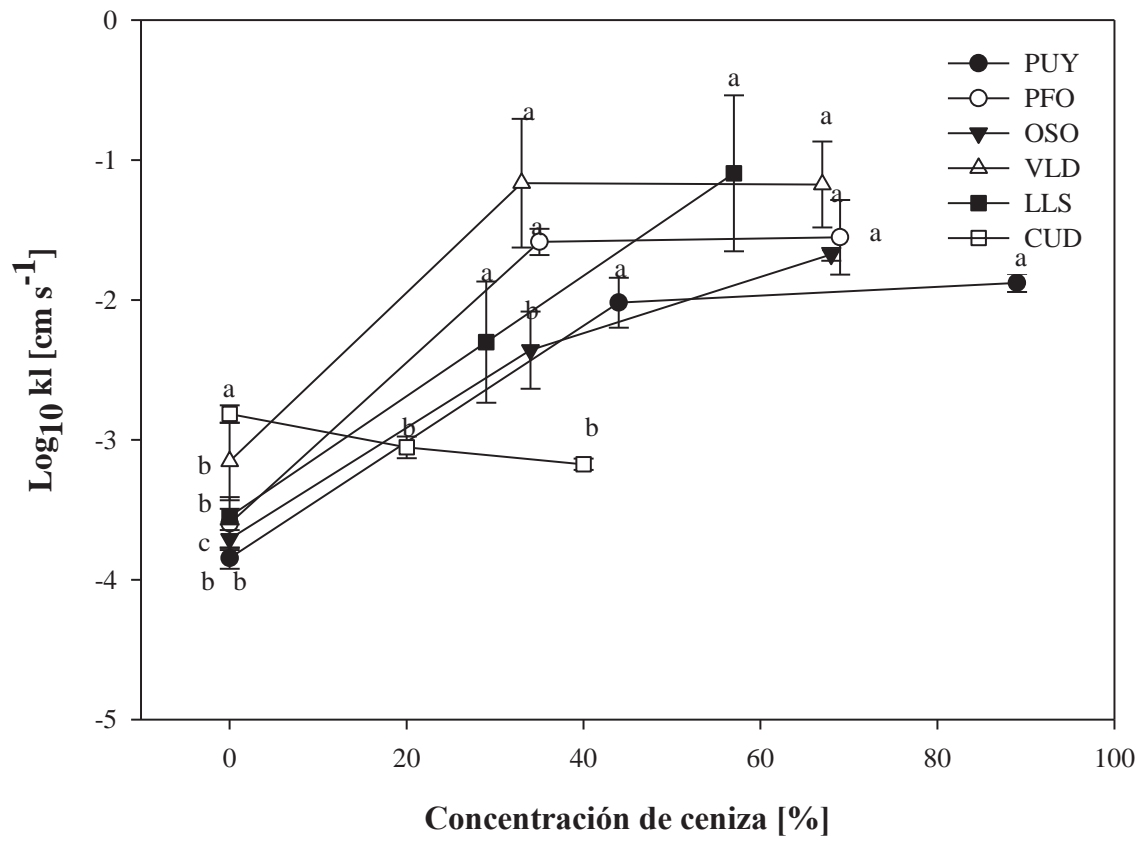

Figura 6. Efecto de la concentración de ceniza/suelo $\left(\mathrm{C}_{\mathrm{c} / \mathrm{s}}\right)$ sobre la conductividad de aire de las series de suelo. Se presentan valores promedio \pm 1 error estándar $(\mathrm{n}=7)$. Letras distintas indican diferencias estadísticamente significativas entre las concentraciones de ceniza para cada serie de suelo $(\mathrm{p} \leq 0,05)$.

Figure 6. Effect of ash/soil concentration $\left(\mathrm{C}_{\mathrm{c} / \mathrm{s}}\right)$ on the air conductivity of soil series. Mean values \pm 1 standard error $(\mathrm{n}=3)$ are presented. Different letters indicate statistically significant differences between the ash concentrations for each soil series ( $\mathrm{p} \leq 0,05$ ).

do de MO con el incremento en la concentración de cenizas (Figura 3) responde, principalmente, al hecho de que las cenizas presentan una cantidad muy baja o nula de materia orgánica produciéndose un efecto de dilución del contenido de esta al ser incorporado el material volcánico (Guevara et al., 2009).

La incorporación de ceniza, así como el aumento de su concentración en el suelo, aumentó el volumen de agua aprovechable para las plantas (poros de agua útil: $\mathrm{PAU}$ ), debido al incremento en el contenido volumétrico de agua a $60 \mathrm{hPa}$ y la reducción del volumen de agua en el punto de marchitez permanente (Figura 4) producto de la reducción proporcional del contenido de arcilla versus el incremento de las fracciones texturales más gruesas (Cuadro 1, Figura 1). Este cambio en la fracción de poros permite que todos los suelos incrementen su volumen de agua disponible para las plantas alcanzado niveles óptimos por sobre el 20\% (Reynolds et al., 2009) valores que son característicos para Andisoles estructurados (Dörner et al., 2013a, b). En ese sentido, cabe destacar el potencial efecto positivo de la incorporación de cenizas volcánicas sobre la calidad física de suelos de textura fina, especialmente, cuando éstos han sido expuestos a manejos que incrementan su densidad aparente y reducen el volumen de agua aprovechable para las plantas (Dörner et al., 2010b, 2013a, b).

Aún cuando se observa que la incorporación de cenizas al suelo produce un incremento en la fracción de po- ros que almacenan agua útil para las plantas (Figura 4), es particularmente importante conocer el nivel de interconexión que presenta el sistema poroso, lo que se puede analizar con la conductividad de aire del suelo (Figura 5). En ese contexto, la incorporación de ceniza provoca un incremento en $\mathrm{kl}$, lo que refleja un incremento de la fracción de poros y de la continuidad que estos tienen para conducir aire. Al homogenizar el suelo y destruir la estructura, los valores iniciales de $\mathrm{kl}$ alcanzados por algunas series de suelo reflejan problemas de compactación ( $\mathrm{kl}<-3,2 \log \mathrm{cms}^{-1}$ según Horn y Fleige, 2009); pero que luego de la incorporación del material de origen volcánico permite alcanzar valores de kl más altos, que aseguran un buen intercambio gaseoso por convección (Dörner et al., 2013a). Sin perjuicio de lo anterior, es necesario profundizar el estudio en este aspecto, ya que como se presenta en la Figura 5, el efecto de la incorporación de ceniza fue negativo para la serie Cudico. Esto se debió a la contracción que sufrió este suelo en los primeros niveles de tensión lo que provocó una reducción inicial del volumen de macroporos, que fue magnificado con el incremento en la concentración de cenizas. En ese contexto, es importante analizar el efecto que tienen los ciclos de mojado y secado del suelo en la estructuración y ordenamiento espacial de los agregados de suelos a los que se le han incorporado materiales de origen volcánico y la respuesta diferencial que pueden tener suelos contrastantes en función de su textura inicial. 
Se pudo determinar, bajo el marco del experimento realizado, que la incorporación de cenizas tiene un potencial efecto positivo sobre la calidad física del suelo. Resulta interesante y, particularmente relevante en el Sur de Chile, continuar este tipo de análisis, pero considerando experimentos de campo, bajo condiciones normales de incorporación de ceniza al suelo (p.ej. en suelos estructurados y no homogenizados como en el presente estudio), que además incluyan estudios de dinámica del agua en el suelo y la respuesta de cultivos/ praderas que son sustentados por ellos (p.ej. Dörner $e t$ al., 2013a; Zúñiga et al., 2015). En ese sentido, la constante actividad volcánica en el Sur de Chile, ofrece un laboratorio natural, tal vez, único a nivel mundial que permite realizar este tipo de estudios, tal como se ha hecho analizando el efecto del cambio de uso de suelo (Ellies et al., 2000; Dörner et al., 2010a), de la intensidad de pastoreo (Dec et al., 2012) y de la labranza(Dörner et al., 2012; Zúñiga et al., 2015) sobre la calidad y funcionamiento físico de este tipo particular de suelos.

\section{CONCLUSIONES}

De acuerdo al experimento realizado, la incorporación de ceniza mejoró el comportamiento físico de los suelos estudiados, considerando sus propiedades físico - hidráulicas. Esto, sin embargo, se presentó en diferente magnitud dependiendo de la textura original del suelo y su diferencia con respecto a la textura de la ceniza incorporada.

El aumento de la carga de ceniza sobre el suelo, provocó un incremento en el contenido de arena y una reducción de la proporción de arcilla. Esto, debido a la naturaleza y granulometría de las cenizas utilizadas. Por otro lado, la densidad real aumentó mientras que el contenido de materia orgánica disminuyó al aumentar la concentración de cenizas en el suelo.

Se observó un incremento en el volumen de agua aprovechable para las plantas (PAU) al aumentar la carga de cenizas lo que está estrechamente relacionado con el cambio en la textura de los suelos (la reducción en el contenido de arcilla provocó una disminución en el contenido volumétrico de agua a $15430 \mathrm{hPa}$, aumentando con ello los PAU).

La conductividad de aire aumentó con la incorporación de ceniza al suelo en todas las series de suelo a excepción de los observado para la serie CUD, lo que siguiere analizar el efecto que tienen los ciclos de mojado y secado del suelo en la estructuración y ordenamiento espacial de los agregados de suelos a los que se le han incorporado materiales de origen volcánico.

\section{AGRADECIMIENTO}

Los autores agradecen al Instituto de Ingeniería Agraria y Suelos por el financiamiento de esta investi- gación. Además, agradecemos la excelente disposición de Don Pedro Soto, dueño del Predio Providencia Lote $\mathrm{C}$, desde donde se recolectaron las cenizas usadas en este estudio.

\section{REFERENCIAS}

Beke, G.J., Sommerfeldt, T.G., 1991. Impact of a subsurface volcanic-ash layer on the properties of a southern Alberta soil with shallow water table. Canadian Journal of Soil Science 71(4), 495-506.

Besoaín, E., 1985. Mineralogía de Arcillas de suelos. Instituto Interamericano de Cooperación para la Agricultura (IICA), San José, Costa Rica.

Besoaín, E., Sepúlveda, G., 1985. Minerales Secundarios, in: Tosso, J. (Ed.), Suelos Volcánicos de Chile, Instituto de Investigaciones Agropecuarias (INIA), Santiago, Chile, pp. 153-214.

Besoaín, E., Sepúlveda, G., Sadzawka, A., 1992. La erupción del volcán Lonquimay y sus efectos en la agricultura. Agricultura Técnica 52(4), 354-358.

Bertrand, S., Fagel, N., 2008. Nature, origin, transport and deposition of andosol parent material in south-central Chile (36-42 $\left.{ }^{\circ}\right)$. Catena 73, 10-22.

Centro de Información de Recursos Naturales (CIREN)., 2003. Descripciones de suelos, materiales y símbolos. Estudio agrologico Xaregión. Centro de información de Recursos Naturales. Santiago, Chile.

Cook, T., Luzio, W., Honorato, R., Galindo, G., Vera, W., Grez, R., 1985. Job description of the Puyehue taxonomic series, in: Beinroth, F.L., Luzio, W., Maldonado, P., Eswaran, H., (Eds.), Proceeding of the Sixth International Soil Classification Workahop. Chile and Ecuador. Part III. Tourguidefor Chile. Sociedad Chilena del Suelo. Santiago, Chile.

Cruz, J., 2011. Efecto de las cenizas volcánicas sobre los suelos de San Luís y La Pampa. Horizonte Agropecuario $\mathrm{N}^{\circ}$ 90. INTA (Instituto Nacional de Tecnología Agropecuaria) Santa Rosa - La Pampa, Argentina.

Chadwick,O.A., Gavenda,R.T., Kelly,E.F., Ziegler, K., Olson,C.G., Elliott, W.C., Hendricks, D.M., 2003. The impact of climate on the biogeochemical functioning of volcanic soils. Chemical Geology 202, 195-223.

Day, P.R., 1965. Particle fractionation and particle size analysis. Methods of soil analysis Part I, in: Black, C.A. (Ed.), Agronomy 9, 545-567.

Dec, D., Dörner, J., Balocchi, O., López, I., 2012. Temporal dynamics of hydraulic and mechanical properties of an Andosol under grazing. Soil and Tillage Research 125, 44-51.

Dörner, J., Horn, R., 2006. Anisotropy of pore functions in structured Stagnic Luvisols in the Weichselien moraine region in North Germany. Journal of Soil Science and Plant Nutrition 169, 213-220.

Dörner, J., Dec, D. Peng, X., Horn, R., 2010a. Effect of land use change on the dynamic behaviour of structural properties of an Andisolin southern Chile under saturated and unsaturated hydraulic conditions. Geoderma 159, 189-197.

Dörner, J., Sandoval, P., Dec, D., 2010b. The role of soil structure on the pore functionality of an Ultisol. Journal of Soil Science and Plant Nutrition 10(4), 495-508.

Dörner, J., Dec, D., Feest, E., Vásquez, N., Díaz, M., 2012. Dyna- 
mics of soil structure and pore functions of a volcanic ash soil under tillage. Soil and Tillage Research 125, 52-60.

Dörner, J., Dec, D., Zúñiga, F., Horn, R., López, I., Leiva, C., Cuevas, J. 2013a. Soil Changes in the Physical Quality of an Andosol under Different Management Intensities in Southern Chile, in: Krümmelben, J., Horn, R., Pagliai, M., (Eds.), Soil Degradation. Advances in Geoecology 42, Catena Verlag GMBH Reiskirchen, Germany, pp. 262-281.

Dörner, J., Zúñiga, F., López, I., 2013b. Short-term effects of different pasture improvement treatments on the physical quality of an Andisol. Journal of Soil Science and Plant Nutrition 13(2), 381-399.

Ellies, A., Horn, R., Smith, R., 2000. Effect of management of a volcanic ash soil on structural properties. International Agrophysics 14, 377-384.

Gaitán J., Ayesa, J., Umaña, F., Raffo, F., Bran, D., 2011.Cartografía del área afectada por cenizas volcánicas en las provincias de Río Negro y Neuquén. Laboratorio de Teledetección, SIG. Instituto Nacional de Tecnología Agropecuaria (INTA), Estación Experimental S.C. de Bariloche, Argentina.

Guevara, A., De la torre, E., Criollo, E., Valencia, L., Gallegos, B., Mena, F., 2009. Evaluación de la influencia de la ceniza volcánica sobre suelos agrícolas aledaños al volcán Tungurahua. Gestión del Riesgo 4(6), 20-23.

Hayashi, K., Okazaki, M., 2002. Effect of Volcanic Fumes from Mt. Oyama, Miyakejima Island, on Atmospheric Deposition, Soil Solution, and Soil Properties in Kumagaya, Central Japan. I. Chemical Properties of Atmospheric Deposition and Soil Solution. Soil Science and Plant Nutrition. 48(3), 401-411.

Hayashi, K., Okazaki, M., 2003. Effect of Volcanic Fumes from Mt. Oyama, Miyakejima Island, on Atmospheric Deposition, Soil Solution, and Soil Properties in Kumagaya, Central Japan. II. Soil Properties. Soil Science and Plant Nutrition. 49(4), 503-511.

Hartge, R., Horn, R., 2009. Die physikalische Untersuchung von Böden. Praxis Messmethoden Auswertung. 4. vollst. Überarbeitete Auflage. Schweizerbart Vorlage, Stuttgart, Germany.

Horn, R., Fleige, H., 2009. Risk assessment of subsoil compaction for arable soils in Northwest Germany at farm scale. Soil and Tillage Research 102, 201-208.

Klute, A., 1986.Methods of soil analysis. Part 1: Physical and mineralogical Methods. $2^{\text {nd }}$ ed. SSSA Book Ser. 5. SSSA, Madison, WI.
Lizcano, A., Herrera, M.C., Santamaría, J.C., 2006. Suelos derivados de cenizas volcánicas en Colombia. Revista Internacional de Desastres Naturales, Accidentes e Infraestructura Civil 6(2), 167.

Maglio, L., Rauque, C.A. 2012. Erupción del complejo Puyehue - Cordón Caulle, efecto sobre la producción acuícola y recomendaciones. http://www.slideshare.net/panchomosquero/volcan-puyehue-cordon-caulle-problematica-postceniza (acceso, s/f)

Reynolds, W.D., Drury, C.F., Tan, C.S., Fox, C.A., Yang, X.M., 2009. Use of indicators and pore volume function characteristics to quantify soil physical quality. Geoderma $152,252-263$.

Riley, C.M., Rose, W.I., Bluth, G.J.S., 2003. Quantitative shape measurements of distal volcanic ash. Journal of Geophysical Research 108(10), 1-15.

Sadzawka, C., Carrasco, M., Grez, R., Mora, M., 2004. Métodos de análisis recomendados para los suelos Chilenos. Comisión de normalización y acreditación. Sociedad Chilena del Suelo, Santiago, Chile.

Sandoval, P., 2009. Curva de retención de humedad, de contracción y permeabilidad de aire en función de distintas densidades aparentes de un suelo Rojo Arcilloso. Tesis Ingeniero Agrónomo, Universidad Austral de Chile. 77p.

Sanhueza, C., Palma, J., Valenzuela, P., Araneda, O., Calderón, K., 2011. Evaluación del comportamiento geotécnico de suelos volcánicos chilenos para su uso como material de filtro en la depuración de aguas residuales domésticas. Revista de la construcción 10(2), 66-81.

Servicio Nacional de Geología y minería (SERNAGEOMIN). 2015. Ranking de los 90 volcanes activos de Chile. http://www.sernageomin.cl/archivos/Ranking-de-Volcanes.pdf (acceso, 21.07.2015).

Shoji, S., Nanzyo, M., Dahlgren, R.,1993. Volcanic ash soil: Genesis, properties and utilization. Developments in Soil Science 21.Elsevier, Amsterdam, Netherlands.

Tosso, J., 1985. Suelos Volcánicos de Chile. Ministerio de Agricultura, Instituto de Investigaciones Agropecuarias. Santiago, Chile.

Townsend, F., 1985. Geotechnical characteristics of residual soils. Journal of Geotechnical Engineering 111, 77-94.

Zúñiga, F., Ivelic-Sáez, J., López, I., Huygens, D., Dörner, J., 2015. Temporal dynamics of the physical quality of an Andisol under a grazing system subjected to different pasture improvement strategies. Soil and Tillage Research 145, 233-241. 
